VI.

Aus der k. k. Universitäts-Ohrenklinik zu Graz (Vorstand Prof. J. Habermann).

\title{
Die Erkrankung des Gehörorgans bei allgemeiner progressiver Paralyse.
}

\author{
Von \\ Dr. Otto Mayer, 1. Assistenten der Klinik.
}

(Mit Tafel I, II)

Von Gehörstörungen bei progressiver Paraly se ist bisher wenig bekannt geworden. Ieh finde z. B. bei v. Krafft-Ebing ${ }^{1}$ ) nur einen Fall von Magn an zitiert, wo Atrophie des Acusticus konstatiert wurde. Es ist dies umso auffälliger, als $\mathrm{v}$. $\mathrm{Kr}$ afftEb ing angibt, dab sich in 4 Proz. der Fälle von Paralyse Opticusatrophie findet und daß auch in der Retina ohne gleichzeitig bestehende Opticusatrophie pathologisehe Veränderungen nachgewiesen wurden (Kle in $\left.{ }^{2}\right)$ ).

Bei den vielfachen Analogien, die sich zwischen dem Verhalten des Optieus und des Acusticus bei den versehiedensten namentlich intrakraniellen Erkrankungen ergeben, wäre also auch bei der Paralyse eine stärkere Beteiligung des Gelörorgans am Erkrankungsprozeß anzunehmen.

Der Klärung dieses Umstandes sind die folgenden Untersuchungen gewidmet.

I. Pathologiseh-anatomische Untersuchungen.

Untersucht wurden 9 Gehörorgane von 5 Paralytikern, in 2 Fällen wurde auch der Hirnstamm histologiseh untersucht.

Die Gehörorgane wurden in die von $\mathrm{Held}$ angegebene und auch von Wittmaak benützte Mischung eingelegt, die aus

1) Krafft-Ebing: Die progress. allgem. Paralyse in Nothnagels spez. Pathologie u. Therapie, IX. Bd. Wien 1894.

2) Klein, Leidesdorf: Psychiatr. Studien 1877, S. 13. 
einer 5 proz. Kaliumbichromatlösung mit 10 Proz. Formol und 3 Proz. Eisessigzusatz besteht.

Die Fixierung in erwähnter Mischung ist der in Formol weit tiberlegen. Außerdem gelingt namentlich die Gieson-Färbung nachher in vorziglich differenzierter Weise. Vor allem aber gestattet sie die von Wittmaak angegebene 0 smierung der Markscheiden, die ich allerdings nur als sekundäre im Schnitt anwandte, die mir aber auch hier gute Resultate gab. Aber es gelingt auch die Markscheidenfärbung nach Wolters sehr gut. Ich betrachte diese von Wittmaak angegebene Methodik als einen bedeutenden Fortsehritt in der Technik der Histologie des Ohres. In bezug auf die näheren Details muB ich auf die betreffende Abhandlung dieses Autors verweisen. $\left.{ }^{1}\right)$

Die Medulla oblongata wurde in fortlaufenden. Serien geschnitten, nachdem sie in Formol fixiert, in Weigerts Beize gechromt, in Alkohol entwässert und in Celloidin eingebettet worden war. Die Schnitte wurden auf Filterpapierblättchen aufgestapelt, jeder 10. Schnitt wurde mit Phenolgelatine-Formol auf dem Objektträger aufgeklebt und nach KulschitzkyWolters gefärbt.

Fall I. S. Georg, 39 Jahre alt, Schreiber.

Diagnose: Paralysis progressiva, Tabes incipiens.

Anamnestisch ist bervorzuheben, daß der Kranke während seiner Militärdienstzeit an Lues erkrankt war und spezifisch behandelt wurde. - Seit November 1905 höhergradige psychische Störungen. Wegen wiederholter Selbstmordversuche im Dezember 1905 an die psychiatrische Klinik abgegeben. Uber Gehörstörungen ist nichts bekannt.

Aus dem Status somatic us der psychiatrischen Klinik erwähne ich folgendes: Die Lidspalten ungleich, rechts weiter als links, die rechte $\mathrm{Pu}$ pille eine Spur enger als die linke. Beide Pupillen reagierend, die linke träger als die rechte. Facialis in allen Ästen symmetrisch. Kniesehnen- und Achillessehnenreflex beiderseits fehlend. Keine schwere Störung des Muskelsinnes und Sensibilität.

Obdnktionsdiagnose (19. Sept. 1906 Dr. Hassmann): Atrophia corticis. Anaemia cerebri, Pneumonia lobularis; dilatatio et hypertrophia ventrucnli sinistri. Amyloidosis bepatis. Infarctes multiplices haemorrhagicae renis sinistri. Colitis membranacea necroticans.

Jinkes Ohr. Paukenhöble, ebenso die zelligen Răume, welche die Labyrinthkapsel umschließen, Antrum und Cellulae mastoideae sind mit zellreichem fibrinösen Exsudat gefunt. Auf der Paukenhöhlenschleimbaut bildet das Exsudat einen dicken Belag, der in den oberflächlichen Schichten aus nekrotischen Zellen besteht, die hier in Fibrinmassen eingelagert sind; in den unteren Schichten beginnt von der Schleimhaut aus bereits eine Organisation des Exsudates. Die Schleimbaut selbst ist dicht infiltriert, verdickt, stellenweise mit kubischem Epithel bedeckt, meist fehlt das Epithel und die Schleimhaut rerschmilzt mit dem Exsudat. Die Schleimhautschicht des Trommelfells ist infiltriert, dieses jedoch nicht perforiert.

1) Wittmaak, Zur histo-patholog. Untersuchung des Gehörorgans etc. Z. f. 0. 51. Bd., II. Heft. 
Die Nische zum runden Fenster ist mit teils organisiertem Exsudat gefüllt, Membrana tympani secundaria nicht entzündet.

Die Uura des inneren Gehörganges ist auffallend zellreich und von Rundzellen durcbsetzt, sie ist aufgelockert, von kleinen Spalten durchzogen, die mit Leukocyten gefült sind, ferner ist sie verhältnismäßig reich an Capillaren. Die oberste Schicht der Dura besteht aus einem zarten Fibrinbelag mit eingelagerten Lenkocyten. Zwischen Dura und Arachnoidea befindet sich ein gröBeres Fibringerinnsel, in welches Leukocyten eingelagert sind. Arachnoidea ebenfalls mit Rundzellen durchsetzt.

Der Ramus cochlearis ist von ungefähr normaler Dicke. An den mit elektrischer Markscheidenfärbung nach Kulschitzky-Wolters gefärbten Präparaten sieht man die Markscheiden vollkommen zerfallen und im Verlauf der Fasern nur mehr in kleinen Segmenten erhalten, die dazwischenliegenden Stücke sind leer. Peripherwärts nimmt die Degeneration der Markscheiden noch weiter zu. Aus den mit Osmium-Pyrogallussäure behandelten Schnitten ergibt sich, daß im Verlauf des Nerven in der Schnecke selbst nur spärliche Myelinreste vorhanden sind; auffallend ist dieses Verhalten im Modiolus, wo die Fasern ganz ungefürbt sind.

Die Ganglienzellen im Spiralganglion sind an Zabl vermindert, man sieht einzelne Lucken; die noch vorhandenen Zellen sind hochgradig degeneriert. Das Protoplasma derselben ist geschrumpft und die Zellen haben hierdurch eckige Konturen. Besonders hochgradig sind diese Veränderungen an der Schneckenbasis.

Die $L$ y $m$ he des peri- und des endolymphatischen Raumes ist uberall gleichmăßig fädig geronnen und mit Gieso on leicht braungefärbt (Fibrin), in dem Gerinnsel sind jedoch keine zelligen Elemente vorhanden.

Die Reissnersche Membran ist straff gespannt. Die Cortische Membran ist etwas geschrumpft und überdacht, in fast normaler Lage die Papilla basilaris. Die Cortischen Pfeiler sind in ihrer Form gut erhalten, in der basalen Windung etwas niedriger, Sinneszellen und Deitersche Zellen fehlen ganz, und von den Stützzellen sind nur mehr niedrige Haufen vorhanden. Besonders hochgradig sind diese Veränderungen in der basalen Windung.

Die Stria vascularis ist etwas verbreitert und sebr reich an Spalten, so daß das Gewebe wie ödematös aussieht, die oberflächliche Epithellage ist stellenweise desquamiert. Die Stria ist gefäbarm, die Capillaren zeigen Endothelwucherung.

Der Ramus vestibularis zeigt ebenfalls bochgradige Degenerationserscheinungen, die peripherwärts zunehmen; in den Verzweigungen in den Maculae und Cristae sind mit Wittmaks Methode nur sparliche Myelinreste nachzuweisen. An Querschnitten der Nervenbündel siebt man fast ganz ungefärbte Bandel neben solchen, die einige Reste von Myelin enthalten. Im Verlauf des Ramus sup. nervi vestibularis befindet sich im Grunde der Fossula superior eine tumorartige Verdickung, welche sich weiter bis zur Macula cribrosasuperior erstreckt. Es besteht diese Anschwellung aus sich durchflechtenden Bündeln von spindelformigen Zellen, mit teils länglichen, teils großen rundlichen Kernen. An seiner oberen Peripherie liegen Nervenfasern und Ganglienzellen, welche dem Ganglion vestibulare superius angehören. Im Inneren des Tumors sind mit Markscheidenfärbung wenige Nervenfasern nachzuweisen.

Die Ganglienzellen des Ganglion Scarpae zeigen Vakuolisierung und Schrumpfung. Die Macula sacculi ist tangential geschnitten. Infolgedessen sind Degenerationserscheinungen nicht sicher nachzuweisen, doch sind die zum Epithel ziehenden Nervenfasern spärlich und die vorhandenen sind degeneriert. Das Epithel der Maculae utriculi zeigt auler den normalerweise sich vorfindenden Vakuolen der Stutzsubstanz große Lucken, in welchen das Epithel fehlt und wo nur einige Kerne mit geringem Protoplasmasaum sich als Uberreste finden. Das Epithel der Cristae ist ebenfalls hochgradig degeneriert; es ist der Raum zwischen Cupula und Basalmembran mit hyalinen Schollen, Kugeln und Kernresten erfült, teils sind 
groBe Lücken vorhanden, in denen sich feine fädige oder körnige Massen befinden; die an das Epithel herantretenden Nervenfasern sind spärlich und die vorhandenen sind hochgradig degeneriert. Doch ist die Substantia propria der Crista nicht atrophiert.

An den Blutgefäßen, namentlich denen im inneren Gebörgang kann man Endothelwucherung und Leukocytheninflitrat in der Adventitia feststellen. Einige haben verkalkte Wände oder sind vollständig obliteriert.

Es fand sich hier also eine fibrinöseitrige Entründung in sämtlichen Mittelohrräumen. Das innere Ohr war jedoch frei von akuten Entzündungserscheinungen. Es war zwar die Lymphe sowoll des endo- als auch des perilymphatischen Raumes geronnen, es kann dies aber nicht als der Ausdruck einer höhergradigen Entzlindung gelten. Vor allem fehlte hierzu die Exsudation von Lenkocyten. Der Nervus acusticus war in seinem Stamm hochgradig, in seinen peripheren Verzweigungen vollkommen degeneriert. Auch die Ganglienzellen und Nervenendorgane, namentlich das Cortische Organ zeigten Degenerationserscheinungen.

Der im Verlauf des Nervus vestibularis gefundene Tumor mun als zellreiches Fibrom angesehan werden. Er verdrängt die Nervenfasern, ohne jedoch eine vollständige Kompression des Nerven herbeizufuhren. Die im weiteren Verlaufe des Astes gefundenen Degenerationserscheinungen sind nicht hochgradiger wie die im Bereiche des Ramus vestib. infer.

Rechtes Ohr. Paukenhöhle, Warzenfortsatz wie links. Dura des inneren Gehörganges zellreicher, ebenso Arachnoidea. Der Stamm des Acusticus atrophisch; an Schnitten, die nach $\mathrm{Kulschitzky-Wolters}$ gefärbt sind, sieht man nur mehr spärliche Markscheiden gefärbt; diese sind dünn und in einzelne Körner und Stäcke zerfallen; diese degenerativen Erscheinungen nehmen peripherwärts zu und man sieht in der Lamina spiralis sowie im Rosenthalschen Kanal nur mehr ganz zarte vereinzelte Fäserchen.

Ebenso zeigen die Nervenzellen des Ganglion spiraledegenerative Erscheinungen; in den unteren Partien ist ein ganz bedeutender Ausfall von Zellen zu konstatieren, der sich durch auffallige liückenbildung kundgibt, die restlichen Zellen zeigen hochgradige Schrumpfung. (Tafel 1/2, Figur 5.)

Die Gebilde des Ductus cochlearis sind ebenso hochgradig verändert. In der basalen Windung ist der Ductus cochlearis auf einen schmalen Spalt verengt dadurch, daß die Membrana vestibularis vom Limbus laminae spiralis, an dessen oberfläche sie angewachsen scheint, quer zur Prominentia spiralis hinüberzieht. (Tafel $1 / 2$, Figur 1.)

Der limbus selbst ist sehr niedrig; von dem nach unten gebogenen Labium vestibulare schlägt sich die geschrumptte Cortische Membran auf einen niedrigen Epithelsaum, den Überrest der Papilla spiralis hinunter, denselben uberdeckend.

In den mittleren Windungen ist die Membrana Reissneri gespannt, sie ist mit dem Limbus verklebt und zleht schräg hinauf zur normalen Inser- 
tionsstelle; die Papilla besteht nur mehr aus platten Zellen, die von der Cortischen Membran uberkleidet werden; in der obersten Windung ist der Limbus laminae spiralis ganz abgeflacht und kaum zu erkennen. Die Papilla spiralis und die Cortische Membran sind daselbst geschwunden.

Die Lamina spiralis ist sehr dünn, die Knochenbălkchen sind atrophiert, namentlich in den oberen Windungen.

Die Stria vascularis ist sehr schmal, gefäliarm, das Ligam. spirale ist atrophisch, namentlich in den oberen Windungen, wo nur einzelne Bindegewebsfäserchen den lückenreichen Raum durchkreuzen.

Auch der Ramus vestibularis ist fast ebenso hochgradig degeneriert wie der Ramus cochlearis.

Das Ganglion vestibulare zeigt Zellausfall und degenerative Veränderung der Nervenzellen. Die Nervenendstellen in den Maculae und Cristae sind hochgradig verändert. Im Bindegewebe der Cristae sind zahlreiche weite Lücken, die Crista ist spitz und erhalt dadurch ein atrophisches Aussehen. Das Epithel ist sehr veränndert, gequollen, von breiten Lücken durchsetzt; ebenso ist das Epithel dor Maculae kaum mehr zu erkennen. Die Nervenfasern zeigen bei elektiver Markscheidenfärbung nur mehr vereinzelte Reste von Myelin.

Sowohl an den Wänden des peri- als auch des endolymphatischen Raumes befinden sich hyaline Massen, offenbar Reste geronnener Lymphe. Auch der Nervus tacialis ist atrophisch.

Die pathologischen Veränderungen an diesem Gehörorgan sind also qualitativ gleich denen des Gehörorgans der linken Seite, nur quantitativ bedeutend boehgradiger. Neben vollkommener Atrophie des Nervus acusticus, sowohl seines Stammes als seiner peripheren Verzweigungen besteht Atrophie der Nervenendstellen und hoehgradige Atrophie und Degenerationen der Ganglienzellen.

Von der Medulla spinalis stand nur das oberste Cervicalsegment zur Verfugung; hier war beiderseits im Gebiet der Hinterstränge ausgedehnte Degeneration nachweisbar. Auf Schnitten durch die Medulla oblongata in der Höhe des Acusticus konnte die Degeneration der Wurzeln des Acusticus mit Sicherheit festgestellt werden.

F a 11 II. F. Joachim, 50 jăhriger lediger Taglöhner.

Klinische Diagnose: Progressive Paralyse.

Der Kranke wurde von der Heimatsgemeinde der phychiatrischen Klinik übergeben am 4. Sept. 1906.

Auszug aus dem $\mathrm{Status} \mathrm{somaticus:} \mathrm{Pupillen} \mathrm{ungleich,} \mathrm{linke} \mathrm{weiter}$ wie die rechte, reagiren träge. Kniereflexe beiderseits gesteigert, kein Fußklonus.

Vorstrecken der Hände gelingt, beim Nachahmen einfacher Bewegungen jedoch ataktisch ansfahrende Bewegungen.

Obduziert in Feldhof am 19. Sept. 1906.

Obduktions-Diagnose: Meningitis chronica, Oedema meningum, Atrophia cerebri. Hydrocephalus chron. int., Euteritis necroticans.

Im ăußeren Gehörgang und am Trommelfell ist nichts Pathologisches $z \mathfrak{u}$ finden.

In der hinteren Umrandung des Ligam. anulare staped. befindet sich eine die ganze Breite des Bandes einnehmende Kalkeinlagerung. Es 
zeigt sich im Hämatose-Eosin-Präparat das Ligament vollkommen durchsetzt von grobkörnigen, tief blau gefärbten Massen, die sich tinktoriell vom Knorpelüberzug des Stapes und des Fensterrandes abbeben. Die vordere Partie des Stapes ist aus dem Fensterrahmen nach aulen gehoben, wodurch das Ringband namentlich vorne stark gedehnt erscheint. Hinten ist die Basis stapedis an normaler Stelle; es scheint also die Basis stapedis um einen hinten gelegenen flxen Punkt, der an der verkalkten Stelle gelegen ist, nach außen gehobelt zu sein. (Tafel 1/2, Figur 4.)

Die Schleimhaut der Paukenhöhle zeigt nirgends pathologische Veränderungen, sie ist dünn, zart, von einem platten, gegen die Tuba zu kubischen Epithel bedeckt.

Die Nische zum runden Fenster ist nicht verändert, ebenso wenig zeigt die runde Fenstermembran (Membrana tympani secundaria) etwas Besonderes. Der Knochen der Labyrinthkapsel ist nirgends, namentlich nicht im Sinne einer Otosklerose verändert.

An der 1 ura des inneren Gehörgangs făllt der bedeutende Zellreichtum auf. Stellenweise verdichten sich die Zellen so, daß es zur Bildung kleiner Infiltrate kommt.

Die Arachnoidea zeigt Endothelwucherungen, ist von Leukocyten durchsetzt und stellenweise liegen auf ibrer Innenseite Fibrinausscheidungen, in denen sich Leukocyten finden, namentlich aber ist der. Nerv an seiner Peripherie von einem Zellmantel umgeben.

Der im inneren Gehörgang liegende Stamm des Nervus acust. ist im ganzen nicht atrophisch. Im zentralen Ende sind große, fast die ganze Nervendicke einnehmende Herde, die sich scharf gegen die Umgebung absetzen, zu sehen. Am unregelmähig gekerbten Rand dieser Herde, ist das Gewebsgefüge gelockert und weist Liücken auf Die Herde sind mit Gieson hellgelb gefärbt, nur einige rote Bindegewebsstränge durchziehen dieselben. Stellenweise kann man die Konturen der Nervenfasern noch unterscheiden, jedoch erscheinen dieselben unregelmäfig konturiert. Kerne sind im ganzen Herd zerstreut, sie gehören Lymphocyten an, größtenteils zeigen sie jedoch Schrumpfung und sind von keinem Protoplasmasaum umgeben. In der Umgebung dieser Herde sind einzelne mit Hämalaun = van Gieson braungefärbte kugelige Gebilde (Corpora amylacea) zu finden. Verfolgen wir den Nerven etwas weiter nach der Peripherie, so können wir auch hier einige kleine Herde von Spindelform, die etwas dunkler braun tingiert sind, konstatieren, im übrigen bestehen in diesen dieselben degenerativen Veränderungen. (Postmortale his tologische Artefakte).

An den mit elect. Markscheidenfärbung behandelten Schnitten (Kulschitzky-Wolters und Wittmaak) sieht man diese Herde sich hell von der geschwärzten Umgebung abheben, nur im Zentrum befinden sich einige schwarze Krummelchen und Faserreste. Bei Gegenfärbung mit Sauerfuchsin werden sie hellgelblich. Weiter peripheriewärts finden sich keine solchen Herde. Betrachtet man die Schnitte mit Markscheidenfärbung, so fällt schon bei schwacher Vergrößerung ein gekörntes Gefüge des Nerven auf und bei starker Vergrößerung ergibt sich als Grund hierfür eine Veränderung der Markscheiden $z \mathbf{u}$ erkennen, die darin besteht, daß auf fadenförmige Verschmälerangen spindelförmige Erweiterungen folgen. Diese Verănderung nimmt im Verlauf der Nervenfasern peripheriewärts an Intensität zu, insoferne als man die fadenförmigen Verbindungsstücke zwischen den verbreiterten oder normalbreiten Partien ganz vermißt, so daßi die Markscheide dadurch in einzelne Segmente zerfällt. Diese Segmentierung kann man durch die Känäle des Mediolus und Tractus spiralis foram. und auch in der Lamina spiralis genau verfolgen.

Die Ganglienzellen des Ganglion spirale scheinen an Zahl nicht vermindert, doch zeigen sie degenerative Veränderungen; sie sind geschrumpft, die Konturen sind nicht rund, sondern eckig. An einigen fehlt der Kern vollständig und die endotheliale Scheide ist von einer krümmeligen, formlosen Masse teilweise erfullt. Auch dieser Zellenrest fehlt manchmal fast vollkommen, so daß nur die leere Hulle sichtbar ist.

Die Gebilde des Ductus cochlearis sind sebr gut konserviert. Die 
Membrana Reissneri ist überall straff gespannt, nur in der Basalwindung leicht gekrümmt. Die Papilla spiralis zeigt in allen Windungen Degeneration der Haarzellen und Deiterschen Zellen bei gut erhaltenen Pfeiler- und Stützzellen. Nur in der basalen Windung sind auch die Pfeiler niedriger, wie eingedrickt. Hier sind auch die Stutzzellen degeneriert: gegen die Spitze zu nehmen alle degenerativen Veränderungen $a b$, so daß in der Spitzenwindung neben gut erhaltenen Pfeiler- und Stutzzellen auch einzelne Haarzellen erhalten sind. An Stelle der fehlenden Sinuszellen liegen teils kugelige, teils krümmelige Massen. Die Cortische Membran ist etwas geschrumpft und von der Papilla abgehoben (Kunstprodukt).

Die Stria vacularis ist von normaler Höhe, der oberflächliche Epithelsaum meist gut erhalten, nur stellenweise mit hyalinen Kugeln besetzt. In den tieferen Schicbten der Stria fällt eine Spalten- und Luckenbildung auf. Die Kapillargefäße zeigen Endothelwucherung. Das Ligament. spirale ist sehr zellarm und sebr linckenreich, namentlich in den oberen Windungen.

Der Nervus vestibularis zeigt ebensolche degenerative Veränderungen wie der Cochlearis nur im geringen Grade. Es ist noch keine Segmentierung zu erkennen, sondern bloß Verdünnung abwechselnd mit Verdickung, namentlich in den Endausbreitungen des Nerven sieht man sehr dünne Fasern, welche stellenweise knotige Verdickungen aufweisen.

Das Epithel der Macula utriculi und sacculi ist nicht höhergradig pathologisch verändert. An den Crista acusticae, namentlich an der des hinteren Bogenganges finden sich im Epithel Lücken. Dieselben befinden sich unmittelbar über der Basalmembran und reichen bis an die Haarzellen beran. Stützzellen fehlen im Bereich der Lücken. Alle Zellelemente sind jedoch unscharf begrenzt und nicht gut erhalten. Bei der großen Labilität dieser Epithelien ist ein sicherer SchluB, ob man die Veränderungen als pathologisch oder postmortal auffassen soll, schwer $z u$ ziehen.

Die Ganglienzellen des Ramus vestibuli sind ebenfalls pathologisch verändert, jedoch in geringerem Grade wie die der Spiralganglien.

Der Nervus facialis zeigt an den mit elekt. Markscheidenfärbung behandelten Schnitten gestreckten Verlauf; seine Fasern sind gut geschwärzt, nur einige unregelmäßig konturiert. An Querschnitten finden sich neben sehr dünnen, auch dicke Fasern, an einigen Fasern fehlt die Markscheide.

Wir finden also außer einer umschriebenen Verkalkung des Ringbandes im Schallleitungsapparat nichts Patbologisches, hingegen degenerative Veränderungen im Ramus cochearis acustici und im Ganglion spirale, geringere im Ramus vestibularis und seinem Ganglion. Im Cortischen Organ Degeneration der Sinneszellen. Die Nervenendstellen an den Maculae und Cristae acusticae nicht deutlich pathologiseh verändert, im inneren Gehörgang Pachy- und Leptomeningitis chroniea.

F all III. F. Anton, 50 jăhriger Bierführer.

Klinische Diagnose: Demeatia paralytica.

Übergeben der Landesirrenanstalt in Feldhof am 11. April 1906.

Anamnestisch ist ein bedeutendes Potatorium hervorzuheben.

Obduziert am 10. Okt. 1906 (Dr. Hassmann).

Obduktion s-Diagn ose: Pachymeningitis hămorrhagica, Encephalitis chronica. Atrophia cerebri, Hydrocephalus chron. internus.

Rechtes Ohr: Paukenhöhle, Trommelfell und Gehörknöchelchen sind normal.

Di e D ura des inneren Gehörganges ist sehr zellreich, stellenweise befinden sich Anhăufungen von Lymphzellen in derselben, angelagert an die 
Arachnoidea befinden sich Fibrinnetze mit eingelagerten Leukocyten, in der Umgebung des Nervenstammes Lymphocyten.

Der Nervus cochlearis ist nicht bedeutend pathologisch verändert; mit electiver Markscheidenfärbung läßt sich geringe Segmentierung konstatieren, namentlich in den Verzweigungen in den $\mathrm{R}$ ose $\mathrm{nth}$ al schen Kanälchen der Schnecke.

Die Nervenzellen im Ganglion cocbleare bieten nur in der basalen Windung Verringerung ihrer Zabl und auch degenerative Veränderungen dar, in den oberen Windungen sind diese gering.

Im Cortischen Organe fehlen die Sinuszellen in der basalen Windung gänzlich, auch der Stützapparat ist defekt, doeh sind die Cortischen Pfeiler deutlich zu erkennen und von normaler Höhe. In den oberen Windungen sind Degenerationen der Deiterscben Zellen, und Sinneszellen deutlich vorhanden, Cortische Pfeiler, Claudius und Hensensche Zellen gut erhalten.

Cortische Membran etwas geschrumpft und von der Papille abgehoben, Reissnersehe Membran etwas in der Ductus cochlearis convex vorgebaucht.

Die Stria vascularis ist etwas breiter wie normal, in den tieferen Schichten wie ödematös, stellenweise ist die oberflächliche Epithellage abgehoben, viel gelbes, körniges Pigment befindet sich im Gewebe In der zweiten Windung befindet sich eine Cyste, welche nur mit einer aus platten Zellen bestehenden Epithelschicht bedeckt ist. Sie sitzt mit breiter Basis auf dem mittleren Drittel der Stria und hat die Form einer Halbkugel; sie ist mit strahligen Massen, die sich mit Hämalaun-Eosin schwach rötlich färben, gefüllt. Das Gewebe der Stria fehlt an der Stelle, so daß die Cyste eigentlich auf der verdickten Innenschicht des Ligament. spirale aufsitzt. (Tafel 1/2 Figur 3.)

Ligamentum spirale gut entwickelt. Der Nervus vestibularis scheint wenig verändert, indem die Segmentierung nicht so deutlich zu sehen ist. Auch in der Aufsplitterung an den Nervenendstellen zeigen die Fasern scharf parallele Konturen und gestreckten Verlauf.

Auch die Nervenzellen des Ganglion vestibulare sind normal.

Von den Nervenendapparaten sind die Maculae normal. An den Cristae ist das Epithel von der Basalmembran abgehoben und in seiner Struktur undeutlich.

Der Nervas facialis zeigt in seinem Querschnitt Faserschwund in einem seiner Bündel, die übrigen scheinen normal.

Die Blutgefäbe im inneren Gebörgang sowie in der Schnecke sind durch Wucherung der Intima in ihrem Lumen verengt, namentlich in der Stria vascularis. Im meat. ac. int. sind einige kleine Gefäße verkalkt und mit ebensolchen Massen angefüllt.

Bei diesem Gehörorgan sind demnach nur geringe degenerative Veränderungen im Cochlearis und Cortisehen Organ nachweisbar. Dagegen Pachymeningitis chronica und Arteriosklerose. Pigmentierung und Oedem der Stria mit Cystenbildung.

Linkes $0 \mathrm{hr}$ : Paukenhöhle, Trommelfell und Fenster normal.

Stamm des Acusticus fehlt (offenbar abgerissen), Dura des innoren Gehörganges zellreich, stellenweise Infiltrate. Fibrinmembranauflagerung sowohl auf der Dura als auch auf der Arachnoidea.

DieVerzweigungen des N e r vus co c h lea e zeigen mit elect. Marksçheidenfärbung sehr deutlich spindelförmige Verdickungen abwechselnd ait fadenförmigen Zwischenstücken, namentlich im Rosenthalschen Kanal.

Nervenzellen des Ganglion spirale nicht merkbar vermindort, jedoch geringgradig degeneriert. Die Gebilde des Ductus cochlearis sma pathologisch verändert. Am auffallendsten ist die Adhäsion der Cortischen Membran an der Oberfiäche der Papille; der Sulcus spiralis incernus, Tunet- 
und Nuëlscher Raum sind stellenweise von einer stärker rötlich tingierten Masse erfüllt, Cortische Pfeiler niedriger, wie eingedrückt. Haarzellen sowie Deitersche Zellen kaum zu erkennen, an ihrer Stelle granulierte Kugeln und Zellen mit stark tingierten Kernen. Die Membrana vestibularis ist mit der Membr. tectoria verklebt, so daß sie erst vom äuBeren Ende der Papille schräg nach oben zieht.

Stria und Ligament. spirale wie rechts, stellenweise Blutungen in den Ductus cochlearis binein. Die Verzweigungen des Nervus vestibularis sind an den Endstellen in geringem Maße degeneriert, ebenso die Epithelien der Membrae und Cristae.

Ganglion nervi vestibul. fehlt. Facialis nicht bedeutend verändert. Gefäßveränderungen wie rechts.

Hier finden sich also neben deutlichen degenerativen Veränderungen im Ramus cochleae, solche geringen Grades im Cortischen Organ. Ferner Pachymeningitis chronica mit Hämorrhagien. Arteriosclerosis, Ödem der Stria usw. wie rechts.

Fall IV. K, Franz, 45 jähriger Bergarbeiter

Klinische Diagnose: Dementia paralytica.

Übergeben der psychiatrischen Klinik 10. Juli 1906. Keine anannestischen Daten.

Aus dem Status somaticus: Pupillen etwas enger, rechte träger reagierend als die linke. Zunge zittert lebhaft, kann nicht vorgestreckt werden. Dabei lebhafte Unruhe, Mitbewegung in allen möglichen Muskelgebieten.

Patellarsehnenreflexe sehr lebhaft gesteigert. Achillessehnenreflexe nicht zu prüfen. Andeutung von Rhomberg.

Obduziert am 19. Juli 1406 (Dr. Hassmann)

Obduktion sdiagn o se: Pachymeningitis haemorrbagica diffusa chron., Meningitis, Hyperaemia cerebri, Oedema cerebri. Encephalomalacia circumscripta, Ependymitis granulosa. Pneumonia lobularis.

Rechtes Ohr: In den zelligen Räumen des Tegmen tymp. befindet sich fibrinöses Exsudat mit Einlagerung von vielen Leukocyten. Dieses Exsudat ist einigen Zellen bereits organisiert. In der $\mathrm{Paukenhöhle} \mathrm{befindet}$ sich kein Exsudat. Die Schleimhaut ist dünn und mit glattem, einschichtigen Epithel bedeckt. Der Boden der Paukenhöhle ist vom Bulbus venae jugularis nur durch ein dünnes Knochenblättchen getrennt; $\mathrm{z}$ wischen der Ampulle des hinteren Bogenganges und dem Bulbus venae jugularis fehlt die knöcherne $Z$ wischenwand an einer stelle ganz, und berühren sich daselbst Periost der Ampulle und die Bulbuswand. Die I ura des inneren Gehörganges ist zellreicher, stellenweise infiltriert; Hämorrhagien befinden sich sowohl zwischen Lura und Arachnoidea, als namentlich $z$ wischen den Verzweigungen des Nerven beim Eintritt in die Knochenkanälchen.

Der Stamm des Cochlearis ist im ganzen nicht verdünnt, in dem interstitiellen Bindegewebe befinden sich reichliche Infiltrate. Bei Osmierung nach Wittmaak tritt geringe Segmentierung der Markscheiden hervor. - Diese Veränderung besteht namentlich im Rosentbalschen Kanal und in der Lamina spiralis.

Die Nervenzellen des Ganglion cochleare sind an Zahl nicht bedeutend verringert, sie zeigen jedoch Schrumpfung und Vacuolenbildung.

Die Papilla spiralis ist ein niedriger Zellhaufen, der mit der Membrana tectoria fest verklebt ist. Die Reissnersche Membran meist nicht straff gespannt, sondern etwas konvex in den Ductus cochl. vorgebancht. Stria vascularis schmal und lang, nicht besonders verändert.

Der Nervus vestibul. enthält neben schön erhaltenen Fasern auch degenerierte.

Im Ganglion vestibulare sind die Nervenzellen meist besser wie im 
Die Erkrankung des Gehörorgans bei allgem. progress. Paralyse. 103

Ganglion cochleare erhalten. Sie zeigen runde Konturen und fullen die Scheide neist voll aus.

In den Aufsplitterungen an den Nervenendstellen sind aber deutliche Verdickungen wechselnd mit fadenförmiger Verdünung zu sehen.

Das Epitbel der Maculae und Cristae ist degeneriert. Die Blutgefäße zeigen hyaline Degeneration und manchmal Verkalkung.

Wir haben also hier Degeneration in der Aufsplitterung der Cochlearis, weniger in der des Vestibularis. Degeneration des Cortischen Organs mittleron Grades. Degeneration der BlutgefäBe; Pachymeningitis chronica haemorrhagiea.

Linkes Ohr: Pankenhöhle, Trommelfell, Fenster normal. Dura des inneren Gehörgangs und Arachnoidea wie rechts.

Ramus cochlearis zeigt einige degenerierte Fasern in seiner Verzweigung in der schnecke, namentlich in der Lamina spiralis.

Die Nervenzellen im. Ganglion cochleare sind an Zahl nicht, oder unbedeutend verringert, zeigen jedoch Degenerationserscheinungen.

Die Papilla spiralis ist etwas niedriger, die Pfeiler sind etwas eingedrückt und der Nuelsche Raum mit hyalinen Kugeln erfüllt. Sinneszellen kaum zu erkennen, Claudiussche Zellen niedriger, in der basalen Windung fehlt das Cortische Organ ganz, Cortische Membran meist abgehoben, Membrana vestibularis schlecht gespannt, unregelmäßig gekrümmt.

Der Ramus vestibularis und das Ganglion vestibulare ist nur in geringem Grade pathologisch. Die Nervenzellen fullen die Scheide vollkommen aus. Doch sind in den Endzweigen der Cristae und auch der Maculae Degenerationserscheinungen nachzuweisen, die Markscheiden werden sehr dünn, färben sich mit Osmium-Pyrogallussäure schlecht und zeigen zwischen fadendünnen Stellen spindelförmige Schwellungen.

Das Epithel der Nervenendstellen ist durch große Vacuolen auf schmale Reste verdrängt. Veränderungen der Meningen und der Gefäße wie rechts.

Zusammenfassung: Geringe Degeneration des Nervus aensticus und der Nervenendstellen, sonst wierechts.

Die Untersuchung der $\mathrm{Medulla}$ oblongata ergibt folgendes:

Anf Durchschnitten in der Höhe der Pyramidenkreuzung läßt sich eine deutlicbe Degeneration des Hinterstranges der rechten Seite konstatieren. Schnitte in der Höhe des Acusticus zeigen Degeneration der medialen und lateralen Wurzeln desselben.

Fall V. R., Franz, 50jähriger verheirateter Kutscher.

Klinische Diagnose: Dementia paralytica.

Der Kranke wurde am 17. September 1906 der psychiatrischen Klinik übergeben. Keine anamnestischen Uaten. Für Gehörstörung keine Anhaltspunkte.

Aus dem Status somaticus: Pupillen miotisch ungleich, lichtstarr, Zunge lebhaft atalktisch zitternd; vorgestreckte Hände zeigen mächtige Unruhe. Kniereflexe wegen ungehöriger Bewegungen des Patienten nicht auslösbar. Gang schwankend, Körperhaltung schlaff.

Obduziert am 11. Oktolver 1906 (Dr. Hassmann).

Obduktionsdiagnose: Meningitis chronica, oedema corticis cerebri, Hydrocephalus chronicus internus.

Rechtes Ohr: Paukenhöhle, Trommelfell, Steigbügelverbindungen, Fensternischen normal. 
D u ra des inneren Gehörgangs etwas zellreicher; der Stamm des Acusticus ist in toto nicht verdannt, in den $Z$ wischenräumen $z$ wischen den Bündeln desselben befinden sich Lymphocyten, ebenso im interstitiellen Bindegewebe des Nerven selbst Infiltrate.

Mit elektrischer Markscheidenfărbung erscheinen die Fasern des Ramus cochlearis segmentiert.

Auch im Ramus vestibularis sind dieselben Zerfallserscheinungen des Myelins, aber in etwas geringerem Grade vorhanden.

In der Sch necke sind die Markscheiden ebenfalls degeneriert. Die Ganglienzellen sind in der Basalwindung verringert, und neben gut erhaltenen befinden sich solche im Stadium des Zerfalls. Nisslfärbung gelingt nicht.

Die Papilla spiralis ist von normaler Höbe, in der basalen Windung fehlen die Sinneszellen und Deitersche Zellen. Clandius- und Hensensche Zellen sind gut erhalten, in den oberen Windungen sind auch Haarzellen deutlich zu erkennen.

Die Cortische Membran ist meist etwas abgehoben und geschrumpft. Die Reissnersche Membran nicht scharf gespannt, meist konvex, in der unteren Windung konkav; in der obersten Windung setzt sie sich nicht an typischer Stelle an, sondern sie zieht senkrecht zum Dach der Scala vestibuli empor.

Die Stria vascularis ist gut ausgebildet und sind die Epithelien dentlich und scharf begrenzt, nur in der basalen Windung zeigen sich zwischen den Zellen Lücken.

Das Ligamentum spirale ist sehr zellarm, luckenreich und in den obersten Windungen auf einen schmalen Streifen reduziert.

Der Ramus vestibuli zeigt geringe Degeneration der Markscheiden.

Die Zellen des Ganglion vestibulare zeigen teils Nisslsche Granula, teils homogenisiertes Protoplasma.

Die Nervenendstellen des Ramus vestib. zeigen geringe Degenerationserscheinungen. Die häutige Ampulle des hinteren Bogenganges zeigt eine auffallende Anomalie, es stülpt sich von ihrer hinteren Wand eine Falte ein, welche in das Lumen der Ampulle frei hineinragt. Da sonst im hãutigen Labyrinth keine Formanomalien zu finden sind, mub ich diese Einstulpung als angeborene Anomalie auffassen.

Der Nervus facialis ist nicht pathologisch verändert.

Es finden sich also anf dieser Seite geringer degenerative Erscheinungen im Acusticus und seinen Endorganen. Im ubrigen sind die Veründerungen qualitativ gleich wie im folgenden linken.

Linke s O br: In der Paukenhöble ist nichts Pathologisches zu finden.

Die Dura des inneren Gebörgangs ist sebr zellreich. Zwischen Dura und Arachnoidea befindet sich an der oberen Wand eine Hämorrhagie, welche die ganze obere Konkavität des Meatus audit. ausfüllt, den Nerv jedoch nicht komprimiert. In der Hämorrhagie sieht man feines fădiges Fibrin, dazwisehen Massen oder Blatkörperchen. Leukocyten in geringer Menge an den Wänden der Blutung.

Auch an anderen Stellen sind kleine Hämorrbagien zwischen Dura und Arachnoidea; längs der Nervenfasern und in den Interstitien finden sich reichliche Leukocyten. Die Blutgefäße zeigen Wucherung der Intima; an $z$ wei mittleren Gefäßen ist die Gefälwand sowie das Lumen von einer Kalkkonkretion ersetzt.

Der Stamm des Nervus acusticus ist im ganzen nicht verdünnt, sein Ramus cochleae zeigt an Schnitten die mit elekt. Markscheidenfärbung nach Wittmaak behandelt sind, dentliche Zeichen won Degeneration, nämlich ausgesprochene Segmentierung der Fasern und bedeutende Vermehrung des interstitiellen Bindegewebes; letzteres kann man besonders an den mit Osmium und Pyrogallus geschwärzten Schnitten und Nachfärbung mit Săurefuchsin, welches das Bindegewebe leuchtend rot färbt, erkennen. Namentlich 
das zur basalen Windung ziehende $\ddot{A}$ stchen des Hömerven ist sehr stark rotgefärbt und ist hier die Segmentbildung besonders ausgesprochen.

Im Modiolus, sowie in der Lamina spiralis läfot sich ein Faserausfall konstatieren und die einzelnen Fasern zeigen hochgradige Zerfallserscheinungen des Myelins.

Uas Ganglion spirale zeigt ebenfalls höhergradige Veränderungen, namentlich in der basalen Windung, wo die Zahl der Ganglienzellen vermindert ist und zwischen normal aussebenden, solche in verschiedenen Stadien des Zerfalles sich finden. AnBerdem ist daselbst eine auffallende Lückenbildung vorhanden. Gegen die Schneckenspitze zu, nehmen diese Veränderungen $\mathrm{ab}$.

Die Gebilde des Ductus cochlearis sind hochgradig degeneriert. Die Reissnersche Membran ist in allen Windungen straff gespannt, nur in den untersten konkav und stellenweise eingerissen, sie ist mit der $\mathrm{Ob}$ ertläche des Limbus verklebt, in einzelnen Windungen auch mit der Cortischen Membran, und zwar bis zur Mitte der restlichen Papilla spiralis, von wo sie schräg zur Seitenwand emporzieht, um entweder an normaler Stelle, oder tiefer an der Stria vascularis zu inserieren.

Das Cortische Organ ist zu einem niedrigen Zellhügel umgewandelt, in dem man die einzelnen Zelloruppen nicht mebr unterseheiden kann. Uber diesen Zellhaufen hinweg zieht, fast mit ihr verklebt, dio verdünnte Cortische Membran.

Die Stria vascularis erscheint niedriger wie normal. Auffallend hochgradig ist jedoch das Ligamentum spirale atrophiert.

Während es in der basalen Windung nur Zellarmut und Lückenbildung aufweist, wird es nach oben zu immer kleiner und fehlt in der Spitzenwindung fast vollstândig, indem die Stria nur mit dünner Bindegewebslage am Periost befestigt ist. (Figur 6.)

In der Schnecke finden sich sehr viel verästelte große $\mathrm{P}$ i g mentzell en.

Das Ganglion vestihulare ist ebenfalls pathologisch verändert. Neben normal aussehenden Zellen liegen solche mit vakuolisierten, geschrumpften Protoplasma, hăufig mit fehlendem Kern, oder es liegen Zellreste in den bindegewebigen Zellscheiden.

Die Fasern des Rarnus vestibularis zeigen auch degenerative Struktur, wie taillenförmige Einschnürung und variköse Erweiterungen in der Aufsplitterung an den Nervenendstellen sind die degenerativen Veränderungen besonders hochgradig.

Die Nervenendstellen im Labyrinth, Maculae wie Cristae sind hochgradig degeneriert. Der Sacculus ist in toto verengt und im Epithel der Macula sind Haar- und Fadenzell $\mathrm{n}$ nicht mehr von einander zu unterscheiden.

Ebensolche Veränderungen finden sich am Epithel der Macula utriculi.

Die Cristae acusticae sind im ganzen atrophiscb, sie sind nicht halbkugelförmig, sondern spitz konisch, im Gewebe derselben sind große Lücken vorhanden. Das kubische Epithel am Rand der Nervenendstelle ist deutlich zu erkennnen, es geht in einen niedrigen Zellhauten über, der die Crista bedeckt und auf welchen keine typischen Zellen mehr berauszufinden sind.

Der Nervus facialis zeigt keine deutlichen Degenerationserscheinungen.

Es findet sich demnach bei normalem Sehalleitungsapparat Degeneration des Nervusacusticus in seinem ganzen peripheren Verlauf, seinem Ganglienapparat und dem Sinusepithel. Die Dura zeigt Erseheinungen von chronisch hämorrhagischer Entzundung; die BlutgefäBe sind sklerotiseb verändert. Insbesondere besteht hochgradige Degeneration des Cortischen Organs, Atrophieder Stria und Schwund des Ligamentum spirale. 


\section{Übersicht der histologischen Befunde.}

Gemeinsam allen untersuchten Gehörorganen sind pathologische Veränderungen im nervösen Apparat, welehe quantitativ auRerordentlich differieren und von beginnender Degeneration bis zu vollkommener Atrophie ansteigen. Ioh fand Veränderungen im Stamm des Acusticus, seinen peripheren Verzweigungen, in seinen Ganglien und Perzeptionsorganen im häutigen Labyrinth. Dazu kamen noeh konstante pathologische Veränderungen im inneren Gehörgang.

Im Stamm des Acusticus im Meatus audit. internus waren erstens herdförmige Degenerationen vorhanden, wie solche in Fall II genauer beschrieben sind. Wenn über die Frage, ob diese herdförmigen, im zentralsten Teile, nahe der Durchsehneidungsstelle des Acusticus gelegenen Degenerationen als pathologisehe oder postmortale, artifizielle, aufzufassen wären, noch bis in die jüngste Zeit bei einigen Autoren Unklarheiten herrschten (Manasse 1)), so ist dureh die experimentellen Untersuehungen Nagers") uber diese Veränderungen der Stab endgültig gebrochen. Die von mir gefundenen Veräuderungen stimmen mit denen, wie sie Nager sehildert, vollkommen uberein, so daß ich hieranf nieht weiter eingehen zu müssen glaube. Ich habe übrigens in den meisten Fällen das zentrale Ende des Acusticus nach erfolgter Fixation abgeschnitten, so daß ich diese Veränderungen selten fand. Jedenfalls müssen wir allen herdförmigen Degenerationserscheinungen im Hörnerven mit Mißtrauen begegnen, da Nager gezeigt hat, daß sich diese Herde peripherwärts von der Zertrümmerungszone noch fortsetzen können.

Zur Erkennung der degenerativen Veränderungen im Nerven leistete mir die Wittmaaksche Methode wie erwähnt, ansgezeiclnete Dienste.

Mit Wittmaak betrachte ich das "Auftreten spindelförmiger Verbreiterungen abwechselnd mit taillenförmigen Verengerungen der Markröhre in den Nervenlängsschnitten" als charakteristisches Zeichen der Degeneration der Nervenfasern. Bei fortsehreitender Veränderung. zerfällt dann die Markseheide in einzelne Segmente, die wieder

1) Manasse: Über chronische, progressive, labyrinthäre Taubheit. Z. f. O. Bd. 52,1 . u. 2. Heft.

2) F. R. Nager: Tber postmortale histologische Artefacte am Nervus acusticus und ihre Erklärung etc. Z, f, O. Bd. 51 , Heft 3. 
Die Erkrankung des Gehörorgans bei allgem. progress. Paralyse. 107

in Teilstiucke zerfallen, so daß schließlich der Nerv bei Markscheidenfärbung wie bestäubt mit feinen sehwarzen Körnchen erscheint, oder daß nur noch stellenweise einzelne zarte Fäserchen erhalten sind.

Alle diese Stadien der Degeneration waren zu beobachten bei Fall I; rechts war der Nerv ganz atrophisch und nur mehr Reste von Myelin zu finden. Links war die Entartung noch nicht so weit fortgeschritten und es war hier hoohgradiger Markscheidenzerfall zu sehen.

In den übrigen Fällen waren die degenerativen Erscheinungen im Stamm des Nerven bedeutend geringer und es waren da Bilder zu sehen, wie sie Wittmaak in seiner Arbeit tiber experiment. degenerative Neuritis des Hörnerven (Z. f. 0.51 . Bd. II Heft, Taf. IV, Fig. 1 n. 2) als Typen anfgestellt hat.

In der peripheren Endausbreitung des Ramus cochlearis waren die Veränderungen der Nervenfasern stets bedeutend hochgradiger wie im Stamm. So fehlten die Fasern im Fall I. R. im Modiolus und der Lamina spiralis nabezu ganz, L. waren sie nur sehr spällich. Dort, wo die Fasern des Stammes sehr geringgradig verändert waren, konnte man an der Peripherie doch schon dentliche Zerfallserseheinungen nachweisen. Der Ramus vestibuli war stets etwas geringer ergriffen wie der Ramus cochleae.

Neben diesen parenchymatösen neuritischen Prozessen sind stets in mebr oder weniger ausgesprochener Weise auch interstitielle zu beobachten gewesen. Diese bestanden in Leakocyteninfiltration im Bindegewebe des Nervus acustieus; eine Bindegewebswucherung konnte ich eigentlich nicht feststellen. Die Leukocytenfiltrate umgaben namentlich auch die feinen Nervenästchen, welche in die basale Windung: eintreten. Ich möchte hier darauf hinweisen, daß die $z u d e n$ basalen $1 \frac{1 / 2}{2}$ Windungen der Schnecke gehörigen Nervenfasern für interstitielle neuritisehe Prozesse besonders disponiert erscheinen, weil sie in fächerförmiger Ausbreitung dem Verlauf des Tractus spiralis foraminosus folgend in den Rosenthalschen Kanal eintreten, während die fur die oberen Windungen bestimmten Fasern in kompaktem Stamme in den Canalis centralis modioli sich begeben. Jene Nervenfasern sind infolge dieses isolierten Verlaufes im Grunde des inneren Gehörorgangs dort einwirkenden Schädlicbkeiten entschieden mehr ausgesetzt als diese. Es scheint mir nicht un- 
walurscheinlieh, daß in manchen Fälen dureh diese topographischen Verbältnissen die vorwiegende Beteiligung der Schneckenbasis bei Cochlearisaffektionen begründet ist.

Ein für diese Ansicht sprechender Fall wurde von Prof. Habermann ${ }^{1}$ ) letzthin in den Beiträgen zur Lehre von der professionellen Sehwerhörigkeit publiziert (Fall III). Es bestand dort bedentende Einengung des Hörfeldes der oberen und unteren Tongrenze, während letzteres Symptom listologiseh durcb Verwachsungen im Schalleitnngsapparat seine Erklärung findet, nimmt Habermann als Grund für den Ausfall der hohen Töne die begleitende Meningitis und Neuritis acustica als Ursache an. Es fand sich nämlich "zwischen Scheide und dem Nervenstamm des Octavus reichlich eitriges Exsudat, das sich stellenweise auch $\eta_{\text {wisehen }}$ den Nervenbündeln eingelagert findet. Besonders ist dies der Fall zwischen den zur Schneekenbasis fülırenden Nerven im Grunde des inneren Gebörgangrs . . .".

Ebenso wie diese akute interstitielle Neuritis wird auch bei der ehronisehen die Schneckenbasis mebr betroffen werden.

Die Ganglienzellen in der Schnecke zeigten ebenfalls in allen Fällen pathologisehe Veränderungen. Sie hielten gleichen Schritt mit denen der Markscheiden. Dort, wo die Zellen höhergradig verändert waren, wie namentlich im Fall I, war auch der Nerv entsprechend degeneriert. - Als Degenerationserscheinungen der Ganglienzellen sind vor allem Sehrumpfung, Vacuolenbildung zu betrachten; dic Zellen werden zackig und das Protoplasma sieht ganz homogen aus, oder das Zellprotoplasma schwindet peripheriewärts so, daß die endotheliale Hülle nicht mehr ganz ausgefüllt wird und der Kern von einem schmäleren Saum von meist granuliertem Protoplasma umgeben ist. Besonders wertvoll ist die Konstatierung der Verringerung der Zellzahl und des Zellausfalles, der sich durch Auftreten von Lückenbildung kundgibt. (Tafel 1/2, Figur 5.)

Diese letzteren Zellveränderungen gleichen denen, die Manasse ${ }^{2}$ letzthin beschrieben und abgebildet hat. In den Fällen, wo die Veränderungen der Ganglienzellen geringgradig waren, versuchte ich die Nisslkörperehen darzustellen, was mir jedoch nicht so befriedigende Bilder gab, dab ich auf Grund dieser weitgehende Sollisse zu ziehen mir erlauben würde; zumal bei Paralyse auch im Facialis typische Veränderungen auf-

1) Habermann, Archiv f. Ohrenheilkde. 69. Bd., S. 116.

2) Manasse, Zeitschrift für Ohrenhlkde. 52. Bd., Taf. IV, Fig. 6. 
Die Erkrankung des Gehörorgans bei allgem. progress. Paralyse. 109

treten, konnte ich die Zellen des Ganglion geniculi nicht zum Vergleich heranziehen, wie Wittmaak ${ }^{\text {) }}$ rät. Da ubrigens aus den experimentellen Untersuchungen dieses Autors hervorgeht, daß sich die „Veränderungen der Nervenzellen in verhältnismäßig kurzer Zeit entwickeln können" und daß, sie reeht häufig bei allen mögliehen sehweren Allgemeininfektionen und Intoxikationen gerade im Ganglion cochleare in den letzten Tagen bzw. Stunden vor dem Tode auftreten", so hat ihre Konstatierung" nicht den Wert, dah aus ihrem Vorhandensein mit Sicherheit Schlitisse gezogen werden könnten. Auch über die Dauer der Erkrankung geben sie uns keinen Aufschluß, da sie sich nach Wittmaak in verhältnismäbig kurzer Zeit entwickeln können.

Doch könnte es sich hier wohl nur um Veränderungen geringen Grades handeln. Jedenfalls brauchen Veränderungen wie sie im Fall I zu sehen sind, längere Zeit zu ihrer Entwieklung. Bei langsam fortschreitenden Prozessen kommt es zu vereinzeltem Zellausfall. Wir müssen demnach die Ganglienzellen in solchen Fällen in verschiedenen Stadien der Rückbildung finden.

Ebenso wie bei Betrachtung der Nervenfasern und Ganglienzellen waren auch im Cortischen Organ aile Stadien der Entartung bis zur vollkommenen Atrophie zu konstatieren.

Wenn ich der durch Alexander ${ }^{2}$ ) und Wittmaak angegebenen Einteilung der pathologiseben Veränderungen im Cortischen Organ in verschiedene Grade folgend, als Veränderungen geringsten Grades die Degeneration und den Ausfall einzelner Sinneszellen bei sonst gut erhaltenem Stützapparat ansehe, so kann ieh mit Rücksicht auf den Einwand, dal es sich bei menschlichen Gehörorganen, die nicht lebenswarm, sondern 10-20 Stunden post mortem eingelegt wurden, wohl leicht um postmortale Veränderungen handeln könnte, hier auf den Wert von Vergleichen der verschiedenen Windungen hinweisen; ferner leisten hier Vergleiche mit dem gleichzeitig eingelegten, nach derselben Methode behandelten Gehörorganen der anderen Seite große Dienste. Ich konnte wiederholt bedeutende Differenzen im Verhalten der beiden Gehörorgane finden und dadureh pathologische Veränderungen mit Sicherheit erkennen.

1) Wittmaak; Weitere Beiträge zur Kenntnis etc. Z. f. $0.53 . \mathrm{Bd}$. 1. Heft, S. 24.

2) Alexander: Zur pathologischen Histologie des Ohrlabyrinths. Archiv f. Ohrenheilk. 56. Bd. 
Die höehstgradigen Veränderungen fand ich im Fall I rechts. (Tafel 1/2, Fig. 1.) Auffallenderweise war gerade hier die Entartung an der Basis geringer wie in der Spitze. Dort feblte die Papilla basilaris vollkommen und es war die Membrana basilaris nur von einem platten Epithel bedeckt; in der basalen Windung konnte man noch einen ganz niedrigen Zellhtigel erkennen. Der Limbus lam. spir. war in der Spitzenwindung vollkommen abgeflacht, nach unten wurde er deutlicher erkennbar, ebenso fehlte oben die Membrana tectoria, unten hedeckte sie den Rest des Cortischen Organes. Hingegen war im Fall V links, wo ebenfalls Veränderungen 3. Grades vorhanden waren, also die Papilla basilaris einen flachen, von der Cortischen Membran bedeckten Zellhaufen bildete, in allen Windungen die Veränderungen ungefähr die gleichen.

Im Fall II waren Veränderungen mittleren Grades vorhanden, die in der basalen Windnng am stärksten ausgebildet waren; dort fehlten Haarzellen und die Deitersehen Zellen, die Claudins'schen Zellen befanden sich in parenchymatöser Degeneration.

Schon bei Veränderungen geringen Grades fand ich die Membrana tectoria manchmal adhärent an der Papille. Wittmakk hat betont, daB diese Fixation ein sicheres Zeichen pathologischer Veränderangen im Cortischen Organe darstelle. Bei den üblichen Präparationsmethoden der Gehörorgane ist die Cortische Membran normalerweise etwas von der Papille abgehoben. Die Fixation der Membran ist daher als pathologisch zu betrachten. Ich glaube dies bestätigen zu können, da ich bei verschiedenen Graden der Degeneration des Cortisehen Organes diese Adhäsion sah. Bei geringgradiger Degeneration des Cortischen Organs fand sie sich im Fall $V$ links. Dort war die Membrana tectoria fest verklebt mit der Oberfläche der Papille und der hierdurch gesehaffene Raum zwischen Suleus spiralis internus und den Pfeilern bis zu den äuberen Stützzellen mit homogener, dunkler tingierter Masse erftillt. Bei hochgradiger Degeneration, wo das Cortische Organ nur mehr einen niedrigen Zellhaufen darstellt, wie in Fall $\mathrm{V}$ links, senkt sich die Cortische Membran hinunter auf diesen Zellbügel und verschmilzt mit diesem zu einer formlosen Masse. Bei Atrophie der Basis, wie im Fall I rechts, war in der obersten Windung aberhaupt von einer Cortisehen Membran nichts mebr wahrzunehmen.

Aber ebenso wie Wittmaak, habe ich gefunden, daf diese Fixation der Membrana tectoria kein konstantes Symptom der 
Die Erkrankung des Gehörorgans bei allgem. progress. Paralyse. 111

Degeneration des Cortisehen Organs ist; so war im Fall I links trotz deutlicher Degeneration die Cortische Membran abgehoben von der Papille, während reehts, wo die Degeneration allerdings viel weiter vorgeschritten war, Fixation der Membrana Corti bestand. Ob aus diesem Verhalten irgendwelche Schlisse auf Alter oder Art der pathologischen Veränderungen im Cortisehen Organ zu ziehen sein werden, müssen weitere Untersuchungen lehren.

Die Stria vasc. bot in mehreren Fällen auffallende Erscheinungen dar. So namentlich im Fall III rechts. (Tafel 1/2, Figur 3.) Dort war die Stria breiter als normal, und es fanden sich zwischen den Zellen Lucken teils an der Basis der Stria, meistens jedoch unter der oberfläehlichen Epithellage. In der Mittelwindung ist an einer Stelle das Epithel halbkugelig vorgebaucht, wodurch eine Zyste gebildet wird. Der Inhalt derselben ist strahlig, stellenweise sind noch unscharf begrenzte runde, stärker tingierte Gebilde, scheinbar Kernreste zu sehen. An der Basis der Zyste befindet sich ebenfalls eine Anzahl von Kernen. Blutgefäße fehlen an dieser Stelle, die Stria ist überhaupt in diesem Falle sehr blutgefaßarm. Das Vas prominens soheint obliteriert. Anffallend ist ferner die Pigmentierung der Stria.

Nach allen diesen Veränderungen glaube ich annehmen zu können, daß es sich hier um eine durch die Gefäßerkrankung: hervorgerufene Staungserscheinung in dem Gewebe der Stria handeln dürfte.

Solche Veränderungen wurden schon von Habermann ${ }^{1)}$ und Alexander') besehrieben. Letzterer fand in einem Falle von kongenitaler Taubheit Atrophie, BlutgefäBarmut der Stria und Cysten derselben und nimmt an, dab es sich um hydropisehe oder hyaline Degeneration der Stria handelt. - Auch Habermann $\left.{ }^{3}\right)$ stellte eine ähnliche Degeneration in einem Fall ron angeborener Taubstummheit fest, ferner bei 4 Fällen von professioneller Schwerhörigkeit. Bei zweien fand er "hochgradige Atrophie der Stria in den oberen Windungen und dazu noch cystenartige Abhebung des Epithels", welche Veränderungen er auf Arteriosklerose zurtukckführt. In den beiden anderen Fällen,

1) Habermann: Archiv f. Ohreilkde. 63. Bd., S. 201.

2) Alexander: Zur Pathologie und pathol. Anatomie der kongenit. Taubheit. Archiv f. Ohrenheilkde. 61. Bd, S. 215.

3) Habermann: Beiträge zur Lehre von der professionellen Schwerhörigkeit. Archiv f. Ohrenbeilkde. 69, Bd, S. 129. 
wo gleichzeitig Tabes bestand, wird die luetische GefäBerkrankung als Ursache der Atrophie der Stria angenommen.

In den vorliegenden Fällen konnte auch ich hühergradige arteriosklerotische GefäBveränderungen finden. Vor allem war die Stria auffallend gefäßarm. Es wäre demnach der Vorgang so zu erklären, daß es infolge der Gefäßerkrankung zu Staungserseheinungen in der Stria kommt. Die Stria wird ödematös, stellenweise cystisch, später wenn die Gefäße obliteriert sind, atrophiert die Stria namentlich in den tiefen Partien vollständig und es bleibt nur die oberfächliche Epithellage zurück.

Wean wir als Funktion der Stria die Sekretion der Endolymphe ansehen, so ist zu erwarten, daß Störungen dieser Funktion anf die Gebilde des Ductus cochlearis, insbesondere auf die Ernährung der Zellen der Papilla spiralis schädlich einwirken werden. Die daraus entstehende Atrophie der Papilla spiralis wäre demnach eine Folge der Gefäßveränderung.

Von pathologischen Veränderungen in den Bogengängen und den Säckehen sind vor allem die Degenerationserscheinungen an den Nervenendstellen anfgefallen; ein alleiniges Befallensein der Schnecke habe ich nie sicher konstatieren können. Es waren immer auch im Gebiet des Nervus vestibularis pathologische Veränderungen entweder deutlich vorhanden oder wenigstens nicht mit voller Sicherheit auszusohließen. Die Epithelien der Cristae acusticae sind sehr leieht veränderlich. Durch Quellung der Stiitzsubstanz werden Vacuolen erzeugt, welche Degenerationen vortäuschen können. Höhergradige pathologische Veränderungen sind dagegen zu erkennen an der Bildung von größeren Lücken im Epithel und körnigem Zerfall desselben. Sobald im Nerven Atrophie eingetreten ist, wie im Fall I rechts, atrophiert auch das Bindegewebe der Crista. So war hier eine auffallende Lüekenbildung zu sehen. An dem Epithel der Maculae waren dieselben Degenerationserscheinungen vorhanden.

Das Ligament. spirale ist ebenfalls in den meisten Fällen patbologiseh verändert. Es zeichnet sich meist dureh große Zellarmut und Liickenbildung aus. Bei Fall V (Tafel 1/2, Figur 6) links fehlt dasselbe in der obersten Windung ganz, in der tieferen ist es hochgradig atrophisch. Im Fall I bestand namentlich rechts hochgradige Atrophie der Substanz des Ligamentes.

An der Dura im inneren Gehörgang konnte man dieselben chronisch entzíndlichen Veränderungen beobachten, wie sie für Paralyse typiseh sind: Infiltrate, Neomembranbildung und Blu- 
tungen. Bei einem Fall füllte die Hämorrhagie die Hälfte des Meatus aus. Ebenso boten Arachnoidea und Pia die Erscheinungen von chronischer Entztindung.

Ehe ich an die Deutung der andiesen Gehörorganen gefundenen Veränderungen herantrete, halte ich es für unerläßlich, die bei Paralyse im Nervensystem auftretenden pathologischen Erscheinungen kurz zu skizzieren, wobei ich der von $\mathrm{A}$. $\mathrm{Cramer}^{1}$ ), respektive $\mathrm{Homen}{ }^{2}$ ) gegebenen Darstellung folge.

Es beginnt die Erkrankung augenscheinlich im Gehirn und ist daselbst „am schwersten und regelmäßigsten die Hirnrinde und das zentrale Höhlengrau" betroffen. Am meisten charakteristisch scheint hier der Schwund markhaltiger Nervenfasern zu sein; aber nicht nur in der Hirnrinde, sondern auch in den uibrigen Teilen des Gehirns, so auch in den Kernen des Hirnstammes "lichtet sich das feine Fasernetz der Kerne".

An zweiter Stelle stehen die Gefäßveränderungen, wie Wucherung des Endothels, hyaline Entartung der Media und Intima, Auswanderung von Leukocyten in den adventitiellen Raum, eventuell Blutung in die perivaskulären Räume und als Folgezustand Ablagerung von Kalk und Pigment in diesen Gebieten.

Erst in dritter Linie nennt $\mathrm{Cramer}$ das Verhalten der Ganglienzellen, die keine, für Paralyse charakteristischen Veränderungen zeigen.

Ferner sind noch zu nennen. Wucherungen der Glia sowie Veränderungen in den Hirnhäuten, die im Beginn der Erkrankung entziindlichen Charakter tragen, der jedoch häufig durch Hämorrhagien ein besonderes Gepräge erhält. Bindegewebsschwarten mit Pigmentanhäufungen bilden die Residuen dieser Veränderungen.

Aber nicht nur das Gehirn, sondern auch das Ruckenmark is t am paralytischen ErkrankungsprozeB beteiligt. Zuerst wurde dies von Westphal gefunden, später von zahlreichen Forschern, zuletzt in gründlicher Weise von Sibellius durch systematische Untersuchung von 25 Fällen studiert. Er fand an den Hintersträngen, Seiten- und Vorderseitensträngen typische Veränderungen; die uns zunächst inter-

1) A. Cramer: Pathologische Anatomie der Psychosen. Handbuch der patholog. Anatomie des Nervensystems 1903. ebenda.

2) E. A. Homen: Rückenmarkserkrankungen bei Dement, paral., 
essierenden Degenerationen der Hinterstränge sind denen der echten Tabes ganz gleichzustellen, sie sind nur quantitativ infolge des frtihen Todes der Paralytikers geringer. In vorgeschrittenen Fällen besteht eine gewisse Proportionalität zwischen den Veränderungen im Rü cken mark einerseits, und denen in den Hinterwurzeln sowie auch in den Spinalganglien andererseits; „die histologischenVeränderungen der genannten Partien sind ganz derselben Natur wie bei Tabes" (E. A. Homen).

Auch in den peripheren Nerven sind bei Paralyse parenchymatöse Veränderungen nachgewiesen worden, die von Pick als zusammenhängend mit dem paralytischen Prozeb, von Furstner als marantische Neuritis aufgefaßt worden sind.

Halte ich die Ergebnisse dieser Forschungen mit meinen Befunden zusammen, so muß ich zum wenigsten im Fall $I$ und IV, in welchen die Degeneration des Acusticus intramedullär verfolgt wurde, die tabische Natur der Degeneration feststellen, umsomehr als die geschilderten pathologischeu Veränderungen im Fall I rechts denjenigen entsprechen, die bei gemeiner Tabes gefunden wurden.

Seit $\mathrm{H}$ abermann als erster und in erschöpfender Weise als Ursache der tabischen Sehwerhörigkeit resp. Taubheit durch genaue pathologisch histologische Untersuchung eines Falles die Degeneration des Acusticus und zwar seiner peripheren Ausbreitung, seines Stammes sowie seiner intra- und extramedullären Wurzeln, nachgewiesen hat, sind von anderen Autoren (Haug, Brahl) weitere Fälle untersucht worden, welche diese Befunde der Hauptsache nach bestätigen. $O b$ bei Tabes und ebenso bei Paralyse die Erkrankung im Ganglion acustici, welches ja einem Spinalganglion entspricht, beginnt, oder ob die Degeneration der Nervenfasern auf ,elektivsystematischem Wege (Schaffer) vor sich geht, das wäre nur an einer Reihe von unkomplizierten Tabesfällen durch genaue Untersuchung des ganzen Acusticus von seinen intramedullären Wurzeln bis zur peripheren Verzweigung zu entscheiden.

Jedenfalls wird man aber bei einem Fall von Paralyse $n \mathrm{u} r$ dann die tabische Natur der Degeneration des Acusticus mit Sicherheit ausschließen können, wenn die Untersuchung der Medulla oblongata negatives Resultat ergibt. Wenn daher Brühl) bei einem Paralytiker Schmiedtaubheit konstatiert

1) Brühl: Zeitschr. f. 0. 52. Bd., Heft 3, S. 243. 
und die im Gehörorgan und dem Cochlearis sich findenden Veränderungen auf diese Ursache zurückführt, so kann dies nur mit Vorbehalt geschehen, da die Medulla oblongata nicht untersucht wurde. Es ist dies umso auffallender, als $\mathrm{Brbh} h \mathrm{l}$ in derselben Abhandlung einen Fall von Taboparalyse mit tabischer Acusticusatrophie beschreibt.

Da auch ich in den abrigen Fällen die Medulla oblongata nicht untersuchte, kann jeh nicht mit vollkommener Sicherheit für die in diesen gefundenen Veränderungen die Tabes verantwortlich machen. Es könnte sich nähmlich in diesen Fällen um neuritische Prozesse handeln, wie sie von Pick, Fürstner und anderen gefuuden wurden und zwar an periph. Nerven und die von Fürstner auf die im Endstadium der Paralyse auftretenden febrilen Erkrankungen, Marasmus usw. bezogen werden.

Man könnte also auch die Veränderungen im Hörnerven als sekundäre, mit dem eigentlichen zentralen paralytischen Prozeß nicht in direktem Zusammenhang stehend auffassen.

Neuerdings hat Wittmaak ähnliche Degenerationen als "senilkachektische Neuritis" und als ,degenerative Neuritis" im Anschluß an versehiedene Intoxikationen und Infektionen genau beschrieben. Habermann hat bereits vor Jahren (Archiv f. 0. Bd. XII S. 37) in seiner Arbeit ubber Nervenatrophie der Schnecke derartige Veränderungen anf sensilen Marasmus usw. zurückgeführt.

Andere Veränderungen im inneren Ohre sind mit Sicherheit anf Arteriosklerose zu beziehen, die ich in den meisten Fällen mebr oder weniger ausgesprochen konstatieren konnte. Gefaßßveränderungen im Gehirn und seinen Häuten sind ja für Paralyse typisch. Die Folgen dieser Gefäßerkrankung zeigten sich unzweifelhaft an der Stria vascularis.

Wenn ich nun an die Zusammenfassung des Gefundenen herantrete, kann ich folgendes feststellen:

1. Es gibt bei progressiver Paralyse degenerative Veränderungen im Acusticus (Stamm, Ganglion, Aufsplitterung), die sicb intramedullär verfolgen lassen und die tabischer Natur sind. Dementsprechend kann umgekehrt fir eine Degeneration des Acusticus bei progressiver Paralyse eine andere Ursache nur dann angenommen werden, - wenn sich die intramedullären Wurzeln als normal erweisen.

2. Daß neben tabischer Atrophie auch marantische 
degenerative Neuritis des Hörnerven vorkommt, ist wahrscheinlich.

3. Es besteht in der Mehrzahl der Fälle höhergradige chronische Entztindung der Gehirnhäute von meist hämorrhagischem Charakter.

4. Auch im Nerven selbst sind interstitielle entzundliche Prozesse zu konstatieren.

5. Ferner: finden sich bei progr. Paralyse Degenerationen im Bereiche des Zirkulationsapparates des inneren Ohres, die auf sklerotischen Veränderungen der Gefäße beruhen und die sekundär eine Atrophie des Cortischen Organs hervorrufen können.

\section{Klinisehe Beobachtungen.}

Im klinischen Symptomenbild der progressiven Paraly se nehmen Gehörstörungen einen geringen Raum ein. Im weitesten Sinne kann man auch Gehörshalluzinationen als solche auffassen, die aber mit einer Erkrankung des Gebörorgans nichts zu tun haben, sondern durch kortikale Reizung entstehen. Im Stadium conclamatum ist uberhaupt schon wegen der vorhandenen Demenz eine Beobachtung feinerer Gehörstörungen ausgeschlossen. Im Stadium prodromorum wurde thergrobe Empfindlichkeit gegen hohe Töne beobachtet $\left(\mathrm{Haug}^{1}\right)$ ). Nach Sand $\mathrm{r}^{2}$ ) treten in $\mathrm{Zwischenräumen} \mathrm{von} \mathrm{3-4} \mathrm{Wochen} \mathrm{Anfälle}$ auf, die sich in nichts von gewöhnlicher Migräne unterscheiden und die mit Überempfindlichkeit allen Gehörseindrücken gegenuber verbunden sind.

Einen hierher gehörigen Fall kann ich als Beispiel fur dieses Symptomenbild anfuhren.

K. Josef, 28jähriger Tischler. Klinis ehe Diagnose: Dementia paralytica; klagt uber Singen in den Ohren und im Kopf seit 1 Jahr. Sein Gehör soll gut sein. Das Klingen in den Ohren entspricht seiner Tonhöhe nach dem $c^{4}$. Zeitweise leidet er an Schwindel.

Die Trommelfelle sind streifig getrubt, eingezogen, der Lichtreflex fehlt.

1) Haug: Krankheiten des Ohres in ihrer Beziehung zu den Allgemeinerkrankungen. 1893. S. 218.

2) Sander: Berl. klin. Wochenschr. 1876. S. 289. 
Die Erkrankung des Gehörorgans bei allgem. progress. Paralyse.

$$
\begin{aligned}
& \text { W. } \left.{ }^{1}\right) \\
& R .=L \text {. } \\
& 1.0 \text { U } 1.0 \\
& +\left(\begin{array}{l}
U_{s} \\
U_{w}
\end{array}\right)+ \\
& 12.0\left(\begin{array}{l}
S t \\
F t
\end{array}\right) 12.0 \\
& 14^{\prime \prime} c_{w v} 15^{\prime \prime} \\
& +24^{\prime \prime} R+25^{\prime \prime} \\
& c_{2}=5^{\prime \prime} c^{c} c^{4}-4^{\prime \prime} c_{2}
\end{aligned}
$$

Die Hörprüfung mit der Uhr sowie mit der Stimme ergibt keine Herabsetzung der Hörschärfe. Bei der Stimmgabelprüfung fällt der stark positive Rinné auf. Die Stimmgabel wurde sowohl in Kopfknochenleitung als auch in Luftleitung in normaler Sekundenanzahl gehört. Auch ist keine Einsehränkung des Gehörs für tiefe Töne vorhanden, so dab also ein Schallleitungshindernis ausgeschlossen ist.

Die Verkturzung der Hördauer bei $\mathrm{c}^{4}$ um 5 " ist zn gering, um daraus mit Sicherheit auf eine Labyrinthaffektion zu schließen, zumal die Knochenleitung für e am Warzenfortsatz normal war, und die obere Tongrenze keine Einschränkung zeigte.

Auffallend war eine große Überempfindlichkeit fur hohe Töne, sowie subjektive Geräusche, von einer Tonhöhe die ungefähr et entsprach. Es ist nun die Frage, wo man die Entstehung dieser Symptome lokalisieren soll, ob sie durch zentrale Reizerseheinungen oder periphere Veränderungen hervorgerufen sind. Bedenkt man, dab im Beginn der Paralyse auch sensible Reizerscheinungen bäufig vorhanden sind, für die tabische oder auch pachymeningitische Prozesse verantwortlich gemacht werden, so könnte man in Analogie dies anch für die Reizerscheinungen in der Gehörsphäre annehmen und eventuell Veränderungen im Acusticusstamm oder seinen Verzweigungen vermuten.

Pathologisch-anatomische Veränderungen, welche derartige Symptome hervorrufen könnten, waren bei fast allen untersuchten Gehörorganen vorhanden. So könnten die ebronischen Entzïn-

1) $W=$ Weberscher Versuch. $U=$ Uhr in Luftleitung. $U_{s}=$ Uhr an der Schlăfe. $U_{i v}=$ Uhr am Warzenfortsatz. $S t=$ laute Stimme. $F l=$ Flüsterstimme. $C_{w}=$ kleine Lucaesche Stimmgabel am Warzenfortsatz (normal 16"). $R=$ Rinne'scher Versuch (normal $36^{* 4}$ ). $C=$ dieselbe Stimmgabel angeschlagen und vor das $\mathrm{Ohr}$ gehalten (normale Hördauer $\left.56^{\prime \prime}\right)$. $C^{4}$ normal $42^{\prime \prime} . \quad H=$ Hörfeld für sămtliche Stimmgabeln in Luftleitung. 
dungserseheinungen der Meningen einen derartigen Reiz auf die Nervenfasern im inneren Gehörgang ausüben. Eine diesbezügliche Beobachtung liegt von Habermann ") vor, der bei einem tabischen Kesselschmied, welcher über Seheinbewegungen in der frontalen Ebene, Schwindel und Erbrechen klagte, eine hochgradige Ansammlung von Leukocyten um den zur hinteren Ampulle führenden Nervenzweig fand. Habermann ist der Ansicht, daß der Reiz, den dieses Infiltrat auf den Nerv ausubte, erwähntes Symptomenbild ausgelöst babe. Zur Unterscheidung, ob der Schwindel zentralen oder peripheren Ursprungs sei, wäre das Vorhandensein von Nystagmus während des Schwindels wichtig. Nach Krafft-Ebing ${ }^{2}$ ) ist bei Paralyse Nystagmus sehr selten, anch bei Tabes kommt gelegentlich Nystagmus von undulierendem Charakter vor; während $U \mathrm{~h}$ to $\mathrm{ff}^{3}$ ) es dahin gestellt sein läßt, ob dieser in Labyrintherkrankungen seinen Grund habe betont B ár ány ${ }^{4}$ ), daB ein rein undulierender Nystagmus bei Labyrintherkrankung niemals vorkommt. Dagegen ist er der Ansicht, daß rythmischer Nystagmus auch bei Tabes auf Erkrankung des inneren Ohres bezogen werden könne.

Bei oben bescbriebenem Fall war ein Nystagmus nicht nachweisbar.

Immerhin ist die Zahl unserer Beobachtungen über Gehörstörungen bei Paralyse eine spärliche, wohl deshalb, weil außer im ersten $\Lambda$ nfangsstadium das Krankheitsgefühl iberhaupt fehlt und die zunehmende Demenz das Bild einer Gehöraffektion unterdrückt. So war nicht einmal im Fall I, wo sich in beiden Schläfebeinen so hochgradige Veränderungen boten, eine Gehörstörung aufgefallen. Man weiß eben nie, was auf schlechtes Gehör und was auf die Unaufmerksamkeit zu setzen ist.

Um jedoch ein ungefähres Bild zu haben von der Häufigkeit der Hörstörungen bei Paralyse, untersuchte ich eine Reihe von Paralytikern in der Landesirrenanstalt Feldhof. Aus dem reichen Material der Anstalt wurden nur solche Kranke aus. gewählt, die noch genügend lucid waren und bei Vornahme der Gehörprüfung mit Bestimmtheit Angaben machten.

1) Habermann: Beiträge zur Lehre von der professionellen Schwerhörigkeit. Dieses Archiv 69. Bd. (2, u. 3. Heft), S. 126.

2) Krafft-Ebing; "Die progressive Paralyse" in Nothnagels spezieller Pathologie und Therapie. S. 48.

3) Uhtoff: Handbuch Grăfe-Sämisch zitiert nach.

4) Bárány: Untersuchungen über den vom Vestibulapparat des Ohres reflektorisch ausgelösten rythmischen Nystagmus. Berlin - Verlag Coblenz. 
Von 10 untersuchten Fällen hatte nur einer subjektive Gehörsempfindungen und die tabrigen behaupteten alle gut zu hören.

Bei der Untersuchung mit Uhr und Flüsterstimme, sowie mit den vorhandenen Stimmgabeln $\mathbf{c}_{4}, \mathbf{c}, \mathrm{C}_{(64)}$ zeigte sich jedoch, dab nur 5 normal börten. Die übrigen hörten die Uhr anf $10-20 \mathrm{~cm}, \mathrm{C}_{(64)}$ wurde gehört, Rinné war positiv, doch war die Knochenleitung verkürzt und die $\mathrm{e}^{4}$ Stimmgabel wurde um 10-15" kürzer gehört wie normal. Bei einem Kranken war außerdem der Rinné auf einer Seite negativ, $C_{(64)}$ wurde daselbst nicht gehört, obwohl der Trommelfells Befund hierfür keine Erklärung bot. Bei der Stimmgabeluntersuchung wurde nur bei wiederholten tibereinstimmenden Angaben Glauben geschenkt, bei widersprechenden Angaben wurde der betreffende Patient nieht weiter gepruft. Die Angaben obiger $10 \mathrm{Kranken}$ sind deshalb insofern als zuverlässig zu betrachten. Wir könnten aus den Gehörprüfungsergebnisse eine Nervenaffektion, bei dem einem Patienten ein einseitiges Schallleitungshindernis, vielleicht StapesAnkylose annehmen.

Wie viel jedoch in diesen Fällen auf Rechnung der psychischen Schwäche des Paralytikers and was auf anatomische Veränderungen im Acustieusgebiet selbst zu setzen ist, ist naturlich nicht sicherzu entscheiden.

Diesen Gehörprüfnngserg̈ebnissen wären die Gesichtsfelduntersuchungen Kornfelds' an die Seite zu stellen, die dieser bei einer großen Zahl von Paralytikern durchführte; er fand nach v. Krafft-Ebing oft sehr beträchtliche konzentrische Einschränkung für weiß, wie auch für Farben, in vorgeschrittenen Fällen bestand mitunter nur zentrales oder fast zentrales Sehen und häufig sektorenförmige Einschränkung. v. Krafft-Ebing ist der Ansicht, daß dieses Symptom fur die Differenzialdiagnose gegen Cerebrasthenie nutzlich sein kann.

Fälle von höhergradigen Hörstörungen bei Paralytikern finden sich in unserem klinisehen Material. So der folgende:

St., Mathias, 55jähriger Farbenreiber: Paralysis progress. incip. - Seit 3 Jahren Singen und Sausen in den Ohren, links mehr als rechts, zunehmende und wechselnde Schwerhörigkeit beiderseits (gegen Abend hört Patient angeblich stets besser);

1) Kornfeld: Zitiert bei v. Krafft-Ebing in Notbnagels spez. Pathologie u. Therapie. IX. Bd. II. Teil, S. 87 . 
dabei öfter Kopfschmerzen, sonst keine Symptome, kein Schwindel, hatte nie Ohrensehmerzen, nie Ohrenfluß, war sonst stets gesund. $W$.

R. $=L$.

0.20 U 0.05

$+\left(\begin{array}{c}U_{s} \\ U_{w}\end{array}\right)+$

$12.2\left(\frac{S t}{F l}\right) 12.0$

$11^{\prime \prime} c_{w} 10^{\prime \prime}$

$+30^{\prime \prime} R+25^{\prime \prime}$

$-7^{\prime \prime} c^{c}-10^{\prime \prime}$

$C_{2}-c^{8} H \overline{C_{2}}-c^{8}$
Rechts: Trommelfell mehr grauweißlich, weniger glänzend.

Links: Stärkere Einziehung des Trommelfells.

Nase: Schleimhaut stark rot, etwas geschwollen, auch die Rachensehleimhant stärker rot.

Nach dieser Gehörpriłfung müssen wir eine Cochlearisaffektion annehmen. Es war Rinne positiv bei starker Verkürzung der Knochenleitung für die Lucaesche Stimmgabel o (vom Warzenfortsatz aus geprüft), die Hördauer für $e^{4}$ in Luftleitung war ebenfalls bedeutend verkürzt, während tiefe Töne gut gehört wurden. Eine Einschränkung des Hörfeldes war weder nach unten, noch nach oben vorbanden.

Bei diesem Falle waren noch keine auffälligen, tabischspinalen Symptome vorhanden, wohl aber im folgenden.

Gs., Johann, 54jähriger Zimmermann. Paralys. progress. - Patient stand bereits vor 7 Jahren mit Symptomen einer beginnenden progressiven Paralyse in Behandlung.

Seit ca. 7 Jahren merkt er langsam zunehmende und angeblich wechselnde Schwerhörigkeit beiderseits mit fortwährendem Geftuhl von Druck in den Ohren, besonders beim Sprechen, konstantes Rauschen, in der Nacht oft Klingen in den Ohren. Seit Beginn seiner Erkrankung vor 7 Jahren zeitweise leichter Schwindel, der seit 1 Jahre ebenfalls stärker und häufiger ist, ebenso die Abnahme der Sehschärfe. Vor 7 Jahren hatte Patient den ersten Schwindelanfall so heftig, daß er angeblich umgefallen ist.

Rhomberg positiv, reflektorische Pupillenstarre, Patellarreflexe fehlend.

$$
\begin{gathered}
R . \bar{W} \\
R .05 \bar{U} 0.05 \\
0.05\left(\begin{array}{c}
U_{s} \\
U_{w}
\end{array}\right) \theta \\
12.0 \stackrel{S t}{ } 120 \\
2.0 F l 2.0 \\
6^{\prime \prime} c_{w} 6^{\prime \prime} \\
+21 R+23^{\prime \prime} \\
-10^{\prime \prime} c^{4}-10^{\prime \prime}
\end{gathered}
$$

Rechts: Trommelfell grau, stärker eingezogen, Randknickung.

Links: Gleich rechts.

Nase: Schleimhaut etwas stärker rot, schleimig-eitriges Sekret in Nase und Rachen in mäßiger Menge. 
Nach dieser Hörpruffung ist ein bedeutenderes Schallleitungshindernis auszuschließen, da keine Einschränkung des Gehörs für tiefe Töne vorhanden war und auch der Rinne beiderseits fast normal positiv ausfiel. Während nämlich die am Warzenfortsatz abgeklungene Lucaesche Stimmgabel e mit 128 Schwingungen in der Sekunde vor das Ohr gehalten, normalerweise $36^{\prime \prime}$ lang gehört wird, wurde sie hier rechts $21^{\prime \prime}$ und links $23^{\prime \prime}$ lang gehört. Hingegen wurde 0 in Knochenleitung vom Warzenfortsatz aus nur $6^{\prime \prime}$ gehört (normal 16"). Das Gehör für hohe Töne hatte ebenfalls gelitten, $\mathrm{c}^{4}$ wurde beiderseits um $10^{\prime \prime}$ kürzer gehört wie normal. Es ist deshalb in diesem Falle das Bestehen einer Cochlearisaffection anzunehmen. Das gleichzeitige Bestehen von Schwindel könnte wohl auch hier für eine Erkrankung des Vestibularis sprechen.

Bei diesem Patienten waren neben der Gehörstörung deutliche tabische Symptome vorhanden, und wir haben es hier offenbar mit einer sogenannten ascendierenden tabischen Paralyse zu tun.

Wie oben erörtert, schließt sich auch an den cerebralen ProzeB typisch eine Erkrankung der Medulla oblongata und spinalis an; daß bei einer bereits entwickelten Dementia die Erkrankung des Gehörorgans keine auffälligen Symptome bieten kann, liegt auf der Hand. Es wird daher eine Gehörstörung nur dann mit Sicherheit zu beobachten sein, wenn der Proze B in der Medulla oblongata bereits Veränderungen gezeitigt hat, bevor noeh die cerebrale Erkrankung voll entwickelt ist.

Es wurde in unserer Klinik ein Fall beobachtet, wo im Anfang der Erkrankung von Seite der Psychiater der Verdacht auf einen Tumor baseos cranii oder pontis bestand, und wo auch eine hochgradige Acusticusaffektion nachweisbar war. Später klärte sich das Symptomenbild im Sinne einer Tabo-Paralyse.

Wenn ich nun zu einem vorläufigen Abschluß meiner Untersuehungen schreite und zur Dentung der klinischen Symptomenbilder die Ergebnisse der histologischen Untersuchung heranziehe, so kann ich folgende Schlüsse ziehen:

1. Die im Anfangsstadium der progressiven Paralyse auftretenden Reizerscheinungen in der Gehörssphäre können auf peripheren Veränderungen im Gehörorgane beruhen, es sind aber zentrale Ursachen nicht ausgeschlossen. 
2. Die Schwerhörigkeit bei progressiver Paralyse ist in den meisten Fällen wohl auf eine Degeneration des Cochlearis zu beziehen, welche am häufigsten tabischer Natur ist; arteriosklerotische und senile (kachektische) Veränderungen werden in den späteren Stadien der Erkrankung sich ausbilden und im klinisehen Symptomenbild nicht hervortreten.

Ich schlieBe meine Ausfuhrungen, indem ich meinem verehrten Chef, Herrn Prof. Habermann, für die Förderung meiner Arbeit wärmstens danke.

Ganz besonders bin ich jedoch Herrn Dr. Heinrich Sterz, Direktor der Landesirrenanstalt in Feldhof, verpflichtet, weil ich seiner Liebenswürdigkeit das Material für diese Arbeit verdanke. Ebenso hatte mit Herr Dr. Hassmann, Ordinarius derselben Anstalt, bei der Answahl des Materials seine Hilfe in der freundlichsten und zuvorkommendsten Weise zur Verfügung gestellt, wofur ich ihm meinen besten Dank sage.

Erklärung der Abbildungen auf Tafel I, II.

Figur 1. Atrophie der Schnecke.

- 2. Degeneratien des Cortischen Organs.

$=3$. Cystenbildung in der Stria vascularis.

$=$ 4. Kalkeinlagerung in das lig. anulare stapedis (Stapesankylose).

= 5. Degeneration der Ganglienzellen des Spiralganglions mit Lückenbildung.

- 6. Degeneration des Cortischen Organs, Atrophie des lig. spirale der Spitzenwindung. 


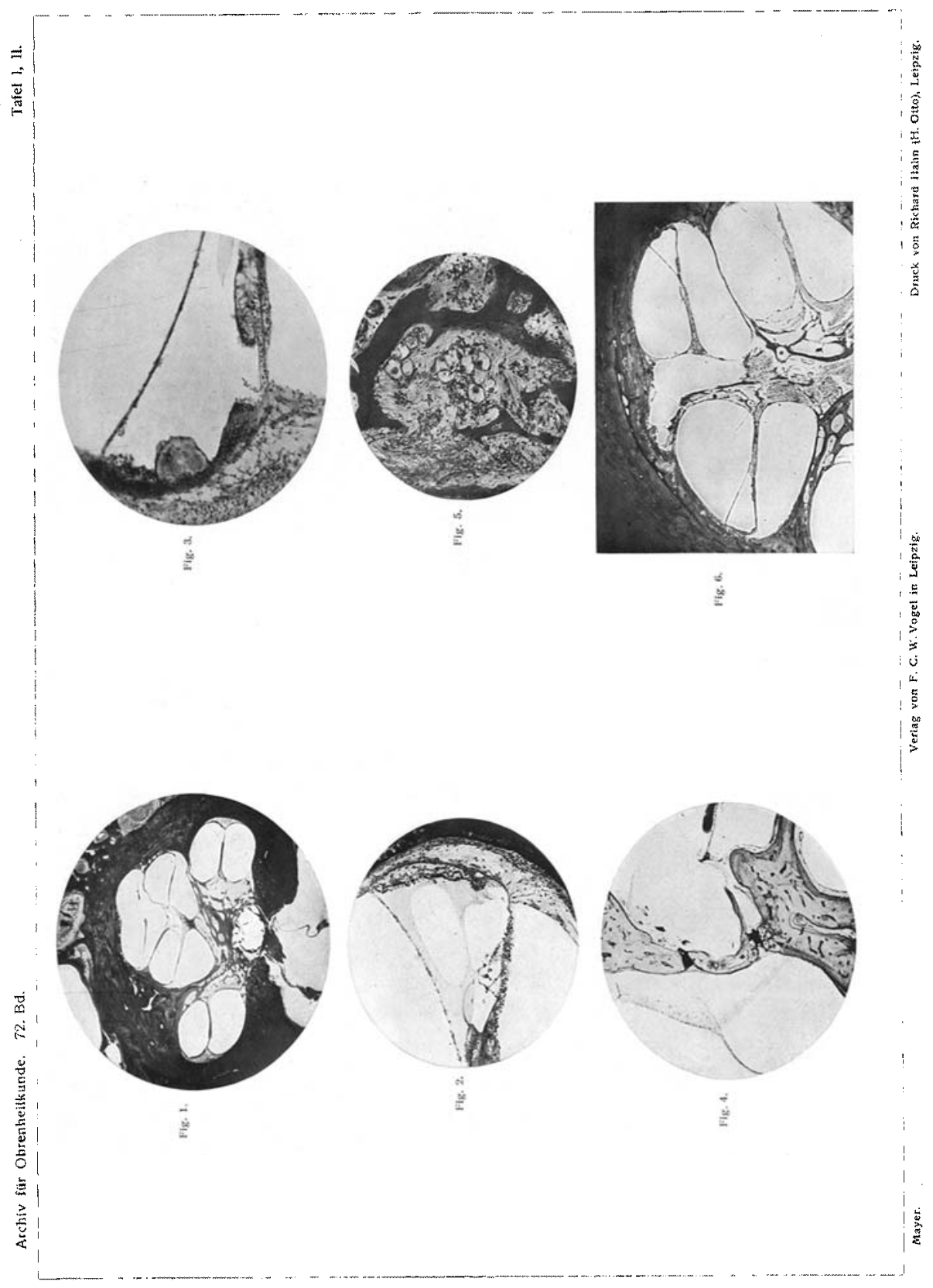

